\title{
O uso de psicofármacos e/ou psicotrópicos: Uma revisão integrativa
}

\author{
Beatriz Soto Boni ${ }^{1}$, Kátia Terezinha Alves Rezende ${ }^{2}$, Fernanda \\ Moerbeck Cardoso Mazzetto ${ }^{2}$, Sílvia Franco da Rocha Tonhom ${ }^{2}$ e \\ Milena Rezende ${ }^{3}$ \\ 1 Egressa do curso de Enfermagem da Faculdade de Medicina de Marília, Brasil | \\ biasboni1806@gmail.com | https://orcid.org/0000-0002-0187-6747 \\ 2 Enfermeira, docente da Faculdade de Medicina de Marília, Brasil | \\ katialvesrezende@gmail.com; fernandamazzetto4@gmail.com; siltonhom@gmail.com | \\ https://orcid.org/0000-0002-9022-2680; https://orcid.org/0000-0001-9276-219X; \\ https://orcid.org/0000-0001-7522-2861 \\ ${ }^{3}$ Médica, Secretaria Municipal de Saúde de Marília, Brasil | milenarezende@hotmail.com | \\ https://orcid.org/0000-0003-2185-1464
}

\begin{abstract}
Resumo: A prescrição de psicofármacos e/ou psicotrópicos aumentou significativamente nos últimos anos, sendo essa classe de medicamento, uma das mais prescritas atualmente. Tratase de uma revisão integrativa da literatura em que a busca foi realizada no BVS, selecionado as bases de dados LILACS e BDENF. Foram analisados 20 artigos de periódicos publicados no período de 2009 a 2019, nos idiomas inglês, português e espanhol. Os resultados mostram que o país de maior produção dos artigos foi o Brasil e indicam que desde o adulto jovem até o idoso são os principais usuários desses medicamentos. Entre os psicofármacos e/ou psicotrópicos mais prescritos estão o Clonazepam, Diazepam e Fluoxetina e os principais prescritores são os clínicos gerais. As situações em que esses medicamentos são prescritos são queixas de ansiedade e insônia. Em relação ao tempo de uso dessas medicações, há prevalência do uso entre três a seis anos. Conclui-se que o uso dessa classe de medicamentos é altamente prevalente, demonstrando a necessidade de Educação Continuada e Permanente em Saúde Mental a esses profissionais, ressaltando o uso racional desses medicamentos.
\end{abstract}

Palavras-chaves: Psicotrópicos; Assistência à Saúde Mental; Prescrições de Medicamentos; Transtornos Mentais; Revisão.

\section{The use of Psychiatric Drugs: An Integrative Review}

\begin{abstract}
The prescription of psychotropic and / or psychotropic drugs has increased significantly in recent years, with this class of medication being one of the most prescribed today. It is an integrative review of the literature in which the search was carried out in the VHL, selecting the databases LILACS and BDENF. Twenty journal articles published from 2009 to 2019 were analyzed, in English, Portuguese and Spanish. The results show that the country with the greatest production of articles was Brazil and indicate that from young adults to the elderly, they are the main users of these drugs. Among the most prescribed psychotropic and / or psychotropic drugs are Clonazepam, Diazepam and Fluoxetine and the main prescribers are general practitioners. The situations in which these drugs are prescribed are complaints of anxiety and insomnia. Regarding the time of use of these medications, there is a prevalence of use between three to six years. It is concluded that the use of this class of drugs is highly prevalent, demonstrating the need for Continuing and Permanent Education in Mental Health to these professionals, emphasizing the rational use of these drugs.
\end{abstract}

Keywords: Psychotropics; Mental Health Assistance; Drug Prescriptions; Mental Disorders: Review.

\section{Introdução}

Atualmente a prescrição de psicofármacos e/ou psicotrópicos é questionada ao redor do mundo, sendo possível destacar que nos últimos anos, o uso desses medicamentos aumentou significativamente, sendo os ansiolíticos a droga mais prescrita mundialmente (Videbeck, 2012). Os psicofármacos são substâncias químicas, naturais ou sintéticas, que quando incluídos no organismo podem causar mudanças de várias maneiras no comportamento mental, excitando, deprimindo ou ocasionando perturbações (Fontana, 2005). 
São indicados para o tratamento de diversos agravos e podem acarretar em dependência física e/ou psíquica, além de contribuírem para a ocorrência de situações adversas aos usuários. Nos últimos anos, observa-se aumento no consumo desses medicamentos, o que pôde ser declarado na Pesquisa Nacional sobre Acesso, Utilização e Promoção do Uso Racional de Medicamentos no Brasil (PNAUM), que explicitou que dentre os 20 subgrupos farmacológicos mais utilizados pelos usuários da rede básica de saúde estão os antidepressivos (Fluoxetina), antiepiléticos e ansiolíticos (Clonazepam), ficando atrás apenas dos fármacos das classes de anti-inflamatórios não esteroidais, anti-hipertensivos e antidiabéticos (SCTIE, 2017).

Em seu estudo, Borges, et al. (2015) concluem que a prevalência de transtornos mentais comuns (TMC) foi de $44,1 \%$. O uso de psicofármacos e/ou psicotrópicos é de $27,1 \%$. Dentro desses $27,1 \%$ do uso desses medicamentos, 30,3\% tem prescrição para uso de 2 ou mais, com prevalência de uso de antidepressivos (45,5\%). Outras patologias associadas: hipertensão $28,5 \%$, diabetes e dislipidemias: $29,3 \%$.

Os estudos mostram maior prevalência de uso de psicofármacos e/ou psicotrópicos por mulheres, tanto na população geral, quanto nos atendimentos na atenção básica. Os transtornos mentais comuns são preditores de pior qualidade de vida em todos os domínios. Destaca-se ainda, alta prevalência de mulheres que reportaram sintomas de ansiedade e depressão. Embora o uso desses medicamentos é altamente prevalente, não foram encontradas evidências documentadas que justifiquem sua prescrição (Borges, Hegadoren e Miasso, 2015).

Braga, et al. (2016). destacam que $52,89 \%$ da amostra fazia uso de benzodiazepínicos isoladamente ou em associação com antidepressivos, em caráter contínuo, ao longo do período de seleção para a coleta dos dados, ou seja, durante seis meses. Desta forma, eles inferem que se trata de uma população com alta probabilidade de dependência provocada por estes fármacos. Schallemberger e Colet (2016) referem que em relação ao grau de ansiedade e dependência, destacam que $47.6 \%$ dos entrevistados são classificados com grau de ansiedade severa, e em relação à dependência, $61.9 \%$ são classificados como potencialmente dependentes. O estudo evidencia que o uso prolongado e inadequado de BZD está se tornando um problema de saúde pública. Destacam ainda, que o crescimento no consumo desses medicamentos demonstra a necessidade de conscientização do uso racional dos mesmos entre os usuários e os profissionais de saúde envolvidos.

O estudo de Rocha e Werlang (2012) destaca uma maior prevalência de uso de psicofármacos e/ou psicotrópicos por mulheres, principalmente antidepressivos, fazendose necessário investigar as razões dessa situação, bem como o planejamento de intervenções na comunidade voltadas a elas. Investigar o perfil de uso desses medicamentos na Atenção Primária a Saúde (APS) faz-se necessário para planejar estratégias de intervenções em saúde mental, relacionadas com a promoção do uso racional dos medicamentos.

O perfil encontrado no estudo e a utilização crônica dos benzodiazepínicos direciona para a necessidade de promover mudanças nas condutas de prevenção, dispensação e utilização desses medicamentos nas unidades da APS, justificado pelo risco de maior tolerância, dependência e efeitos colaterais desses medicamentos na população em estudo (Silva, et al., 2015).

Identifica-se a necessidade de mudar o paradigma dos serviços de saúde para que comunidade, profissionais de saúde e gestores reconheçam que os TMC representam parte significativa da APS. As unidades desse nível de atenção são essenciais para avaliar a saúde mental de seus usuários, e esses profissionais devem ser capacitados para desenvolver o cuidado na perspectiva da integralidade às pessoas que possuem TMC (Borges, et al. 2015). 
Para Rocha e Werlang (2012) a seleção dos psicofármacos e/ou psicotrópicos nas relações de medicamentos essenciais, baseada em critérios epidemiológicos, a elaboração de protocolos clínicos para a utilização dos mesmos e a capacitação em saúde mental para os profissionais que atuam na APS, são imprescindíveis para a promoção do cuidado na direção da integralidade.

Sabe-se que o uso prolongado dos psicotrópicos, mesmo em doses adequadas, pode causar dependência psíquica e física, tolerância e síndrome de abstinência. Assim, recomenda-se aos médicos, boas práticas de atenção à saúde, evitando o uso indiscriminado ou impreciso destes fármacos, bem como divulguem a vasta gama de efeitos colaterais aos seus pacientes, de modo a melhorar a sua qualidade de vida (Braga et al., 2016).

Esses autores apontam que há necessidade de programar ações nos três níveis de atenção à saúde, com o intuito de melhorar a qualidade de vida das pessoas e, consequentemente diminuir o consumo indiscriminado destas classes de medicamentos.

Enquanto a atividade de prática profissional e estudante do $2^{\circ}$ ano de enfermagem experiencia-se, na Unidade Educacional Eletiva (UEE) em uma Unidade Básica de Saúde (UBS) de um município de pequeno porte, localizado no estado de São Paulo. Durante o desenvolvimento dessa Unidade se observa grande parcela da população utilizando psicofármacos e/ou psicotrópicos. Destaca-se que durante as pós consultas de enfermagem evidencia-se que de 20 pacientes atendidos por período, pelo menos 50\% deles saiam da consulta médica com prescrição desses medicamentos.

Diante desse cenário, questiona-se qual o perfil dos usuários no contexto da atenção primária, que utilizam psicofármacos e/ou psicotrópicos, encontrados na literatura, em língua inglesa, espanhola e brasileira?

Assim, essa pesquisa objetiva analisar o perfil do uso de psicofármacos e/ou psicotrópicos, no contexto da atenção básica, reportados na literatura mundial.

\section{Metodologia}

O presente estudo trata-se de uma Revisão Integrativa de Literatura (RIL). A RIL é um método que tem como função sintetizar os resultados obtidos em pesquisas sobre um tema, de maneira sistemática, ordenada e abrangente. É denominada integrativa pois fornece informações amplas sobre um assunto/problema, constituindo, assim, um corpo de conhecimento (Ercole, et al., 2014).

Buscando seguir o rigor metodológico da RIL, conforme Ganong (1987) e Whittemore e Knafl (2005), seguiu-se as seguintes etapas:

A primeira etapa é a identificação do problema e a seleção da questão de pesquisa. Nesse sentido, diante da vivência nos cenários de formação acadêmica, identificou-se que grande parte da população faz uso de psicofármacos e/ou psicotrópicos, sendo assim, questionou-se qual o perfil dos usuários que utilizam esses medicamentos, em quais situações e problemas de saúde são prescritos, quais são os mais prescritos, tempo de uso e quem é o principal prescritor?

$\mathrm{Na}$ segunda etapa, a seleção dos artigos foi por meio dos seguintes descritores: antidepressivos, ansiolíticos, psicofármacos, psicotrópicos, psicoativos, consumo indevido, indiscriminado, inadequado e abusivo, medicamentos, remédios, atenção primária, estratégia de saúde da família e unidade básica de saúde; nas bases de dados BDENF e LILACS, nos idiomas português, inglês e espanhol, no período de 2009 até 2019

$\mathrm{Na}$ terceira etapa, identificou-se 54 artigos que após leitura dos títulos e resumos, foram excluídos 34, considerando como critérios de exclusão: artigos que não se enquadraram na temática em questão, como por exemplo, artigos que trazem opções de terapêuticas naturais ou que trazem pesquisas de um medicamento específico, artigos que estavam sendo repetidos, além de teses, dissertações e artigos de revisão integrativa. 
Como critérios de inclusão: artigos que respondem à pergunta de pesquisa, publicados em português, inglês e espanhol em um período de 10 anos. Sendo assim, finalizou-se com 20 artigos para compor o corpus da análise.

Para finalizar, na quarta etapa decorreu-se a análise dos resultados, partindo da identificação de convergências e divergências entre eles. Assim, obteve-se as seguintes temáticas que serão abordadas a seguir: perfil dos usuários que utilizam psicofármacos e/ou psicotrópicos; situações/problemas de saúde em que os psicofármacos e/ou psicotrópicos são prescritos; os psicofármacos e/ou psicotrópicos mais utilizados; tempo de uso de psicofármacos e/ou psicotrópicos e formação profissional dos prescritores.

\section{Resultados}

A partir da busca realizada, considerando os objetivos propostos, apresenta-se o perfil dos usuários que utilizam psicofármacos e/ou psicotrópicos, as situações e problemas de saúde em que são prescritos, os mais prescritos, o tempo de uso desses medicamentos e a especialidade médica que os prescreveu.

\subsection{Perfil dos Usuários que Utilizam Psicofármacos e/ou Psicotrópicos}

Dentre os artigos analisados, identifica-se que todos os autores trazem o perfil dos usuários de psicofármacos e/ou psicotrópicos, indicando que a maioria são mulheres.

Ramon et al. (2019), Medeiros Filho et al., (2018), Silva et al. (2016), Schenkel e Colet (2016), Schallemberger e Colet (2016), Borges et al. (2015), Silva et al. (2015), Telles Filho et al. (2011) revelam que os pacientes em estudo possuem baixa escolaridade.

Em relação à ocupação, Ramon et al. (2019) relatam que a maior parte são desempregados. Medeiros Filho, Azevedo, Pinto e Silva (2018). evidenciam que a maioria são trabalhadores do lar. Schenkel e Colet (2016), Mezzari e Iser (2015), Baldoni et al. (2013), Telles Filho et al. (2011) referem porcentagem maior de aposentados. Borges, Hegadoren e Miasso (2015) e Borges et al. (2015) indicam, apenas, a prevalência dos entrevistados que trabalham com inserção formal e/ou informal.

Quanto ao número de pessoas com quem os usuários de psicofármacos e/ou psicotrópicos residem, Ramon et al. (2019) mencionam até três moradores; Borges, Hegadoren e Miasso (2015) declaram com até quatro; e Medeiros Filho et al. (2018) relatam que os usuários residem com cônjuge e filhos.

Medeiros Filho et al. (2018), Silva et al. (2016), Schenkel e Colet (2016), Schallemberger e Colet (2016), Borges, Hegadoren e Miasso (2015), Mezzari e Iser (2015), Borges et al. (2015), SILVA et al. 2015 e Nordon, Akamine, Novo e Hübner (2009) evidenciam que os usuários são em sua maioria casados e Telles Filho et al. (2011) identificam que em sua pesquisa uma grande porcentagem dos usuários são solteiros.

Bueno, Almeida e Rocha, (2016), Schenkel e Colet (2016), Schallemberger e Colet (2016), Mezzari e Iser (2015) relatam que os usuários são idosos. Os demais autores apontam que os usuários desses medicamentos pertencem aos diferentes ciclos de vida, adulto jovem, adulto e idoso.

\subsection{Situações/Problemas de Saúde em que os Psicofármacos e/ou Psicotrópicos são Prescritos}

Dentre os artigos analisados, identifica-se que Medeiros Filho et al. (2018), Bueno, Almeida e Rocha (2016), Braga et al. (2016), Schallemberger e Colet (2016), Borges et al. (2015), Rocha e Werlang (2013), Baldoni et al. (2013) e Telles Filho et al. (2011) não apresentam as situações em que os psicofármacos e/ou psicotrópicos foram indicados. 
Ramon et al. 2019, Silva et al. (2016), Silva et al. (2015), Sánchez Ricardo e Hérnandez Gárciga (2010), Nordon et al. (2009), Mezzari e Iser (2015) apontam que as queixas mais referidas que levaram ao uso dos psicofármacos e/ou psicotrópicos foram ansiedade $(42,10 \%)$ e insônia $(35,08 \%)$. Ramon et al. (2019) também indica a dor como situação em que são prescritos e Nordon et al. (2009) cita a depressão, convulsões/epilepsia e luto.

Para Pereira et al. (2012) os transtornos neuróticos relacionados ao estresse totalizaram 16,4\% dos diagnósticos no Ambulatório de Saúde Mental e transtornos mentais e comportamentais em razão do uso de substâncias psicoativas $(25,8 \%)$, seguidos de diagnósticos de Esquizofrenia e Transtornos Delirantes (21,7\%) no Centro de Atenção Psicossocial (CAPS)

Yoneyama, Maruiti e Esteves (2016), Lima e Fleck (2011) e Mezzari e Iser (2015) evidenciam que dentre os diagnósticos, o mais comum está a Depressão. Para além dessa, Yoneyama, Maruiti e Esteves (2016) menciona a insônia não orgânica.

Segundo Olivera (2009) os psicofármacos foram empregados em casos de Demência, Distúrbio do Sono, Dependência de Álcool, Distúrbio do Pânico e Transtorno de Ansiedade Generalizada; Transtorno de Humor, dos quais correspondem a Depressão Crônica, episódio Depressivo Grave e a Transtorno Afetivo Bipolar.

Mezzari e Iser (2015) indicam ainda outras situações em que os psicofármacos são empregados, como Esquizofrenia, Epilepsia e Transtorno de Pânico.

A Hipertensão Arterial Sistêmica (HAS) também foi mencionada como situação em que se prescreveu os psicofármacos e/ou psicotrópicos segundo Sánchez Ricardo e Hérnandez Gárciga (2010). Esses autores também citam outros motivos, mas não os explicitam.

\subsection{Os Psicofármacos e/ou Psicotrópicos Mais Utilizados}

Dentre os artigos analisados, identifica-se que Medeiros Filho et al. (2018), Yoneyama et al. (2016), Borges et al. (2015), Baldoni et al. (2013), Pereira et al. (2012), Lima e Fleck (2011) e Olivera V (2009) não apresentam os psicofármacos e/ou psicotrópicos mais utilizados.

Ramon et al. (2019), Silva et al. (2016), Braga et al. (2016), Schallemberger e Colet (2016), Mezzari e Iser (2015), Borges et al. (2015), Silva et al. (2015), Rocha e Werlang (2013), Telles Filho et al. (2011) e Nordon et al. (2009) relatam que o Clonazepam está entre os benzodiazepínicos mais utilizados.

Ramon et al. (2019), Bueno, Almeida e Rocha (2016), Rocha e Werlang (2013) e Borges et al. (2015) identificam ainda o uso da Amitriptilina. Schenkel e Colet (2016) e Ramon et al. (2019) relatam o uso do Citalopram.

Silva et al. (2016), Bueno, Almeida e Rocha (2016), Schallemberger e Colet (2016), Mezzari e Iser (2015), Borges et al. (2015), Silva et al. (2015), Telles Filho et al. (2011), Rocha e Werlang (2013), Nordon et al. (2009) e Sánchez Ricardo e Hérnandez Gárciga (2010) relatam o uso do Diazepam.

Bueno, Almeida e Rocha, 2016, Schenkel e Colet (2016), Borges et al. (2015), Rocha e Werlang (2013) e Braga et al. (2016) ainda mencionam que a Fluoxetina é um dos psicofármacos com maior prevalência de prescrição. Schenkel e Colet (2016) indicam a Sertralina. Mezzari e Iser (2015) e Telles Filho et al. (2011) relatam o uso de bromazepam. Sánchez Ricardo e Hérnandez Gárciga (2010) mencionam o uso do Clorodiazepóxido. Borges et al. (2015) indicam o Escitalopram.

Telles Filho et al. (2011) e Rocha e Werlang (2013) relatam o uso de Alprazolam. Telles Filho et al. (2011) apresenta o uso de Lorazepam e Midazolam. Rocha e Werlang (2013) menciona ainda o uso de Carbamazepina. Silva et al. (2015) refere o uso de Nitrazepam. 


\subsection{Tempo de Uso de Psicofármacos e/ou Psicotrópicos}

Dentre os artigos analisados, identifica-se que Bueno et al. (2016), Schenkel e Colet (2016), Yoneyama, et al. (2016), Borges et al., (2015), Borges et al. (2015), Rocha e Werlang (2013), Baldoni et al. (2013), Pereira et al. (2012), Telles Filho et al. (2011), Lima e Fleck (2011), Sánchez Ricardo e Hérnandez Gárciga (2010) e Olivera V (2009) não apresentam o tempo de uso dos psicofármacos.

Ramon et al. (2019), Braga et al. (2016) e Nordon et al. (2009) apontam que a maioria dos pacientes utilizam os medicamentos há menos de um ano. Schallemberger e Colet (2016) relatam que o uso desses medicamentos é maior do que um ano, não delimitando o tempo.

Medeiros Filho et al. (2018), Silva et al. (2016), Silva et al. (2015), Mezzari e Iser (2015) apontam média de três a seis anos.

\subsection{Formação Profissional dos Prescritores}

Dentre os artigos analisados, identifica-se que Bueno et al., (2016), Schenkel e Colet (2016), Braga et al. (2016), Schallemberger e Colet (2016), Yoneyama, Maruiti e Esteves (2016), Borges et al. (2015), Borges et al. (2015), Rocha e Werlang (2013), Baldoni et al. 2013, Pereira et al. (2012), Telles Filho et al. (2011), Lima e Fleck (2011), Sánchez Ricardo e Hérnandez Gárciga (2010) e Olivera V (2009) não apresentam a especialidade do médico que prescreveu esses medicamentos.

Ramon et al. (2019) e Medeiros Filho et al. (2018) apontam que o principal prescritor dos psicofármacos são os psiquiatras.

Silva et al. (2016), Mezzari e Iser (2015), Silva et al. (2015) e Nordon et al. (2009) apontam que o principal prescritor dos psicofármacos são os clínicos gerais.

Mezzari e Iser (2015) e Nordon et al. (2009) mencionam que além dos clínicos gerais, a segunda especialidade médica que mais prescreve psicofármacos e/ou psicotrópicos são os psiquiatras e em terceiro lugar outras especialidades, não mencionando quais.

Ramon et al. (2019) indica que além dos psiquiatras, a segunda especialidade médica que mais prescreve os psicofármacos e/ou psicotrópicos são os neurologistas e em terceiro lugar os clínicos gerais, seguido por outras especialidades, não mencionando quais.

\section{Discussão}

Passos Neto et al. (2020) também identificaram as mulheres como principais usuárias dos psicotrópicos, a maioria eram casadas, predomínio de aposentadas e a maior parte residiam com até quatro moradores na mesma residência. Similarmente, Prado, Francisco e Barros (2017) observaram que a maioria das pessoas que utilizavam esses medicamentos era casada.

A investigação de Rodrigues et al (2020) evidenciaram que 55\% dos adultos e 50\% dos idosos referiram fazer uso de antidepressivos. Os ansiolíticos também apresentaram uma elevada taxa de uso, principalmente em idosos. No que tange à idade, a média encontrada foi de 39,6 anos nos adultos e de 70,2 nos idosos e a prevalência do uso de psicotrópicos observada na população adulta e idosa brasileira residente na Zona Urbana foi de $8,7 \%$.

A maior utilização dos psicotrópicos foi observada em mulheres e com os que referiram maior número de doenças crônicas. As regiões com menor prevalência de utilização desses medicamentos foram a região Norte e Nordeste, enquanto as que apresentaram maior prevalência de uso foram as regiões Sudeste e Sul (Rodrigues et al., 2020)

Oliveira et al. (2021) apontaram que um em cada cinco pacientes que retiraram medicamentos do componente básico do município utilizava psicofármacos, sendo em sua maioria mulheres. 
Em relação à escolaridade, Rodrigues et al. (2020) concluem que há prevalência de usuários com zero a oito anos de estudos.

Passos Neto et al. (2020) referem a prevalência de usuários que não informaram o principal motivo para o uso desses medicamentos, seguidos pelo uso por motivos de insônia e nervosismo, sendo os motivos menos prevalentes, como: agitação, taquicardia, entre outros.

A justificativa do uso do psicotrópico, começou centrada no corpo, com ênfase para dor ou necessidade fisiológica como choro, nervosismo, impossibilidade de fazer coisas que antes fazia, pensamentos ruins, preocupações, dificuldade/impossibilidade de dormir, dores, isolamento, angústia e dores de cabeça (Molck, Barbosa e Domingos, 2021).

A investigação de Filardi, Mendonça e Oliveira (2021) apontou que o psicotrópico tornouse necessário quando os problemas vividos passaram a ser reconhecidos com um alto grau de dificuldade, que ultrapassava a capacidade pessoal para a sua resolução. Esses problemas enfrentados no cotidiano do trabalho, da família, das relações amorosas e dos estudos, desencadearam sentimentos de raiva, ansiedade, estresse, tristeza e angústia, os quais foram atravessados pela proximidade e a vivência de situações muito graves como a pobreza e a violência. A percepção, quando se está vivendo essas emoções, é que a vida está fora de controle, há o medo subliminar de enlouquecer ou de que as reações frente ao problema não sejam normais.

Destacou-se uma prevalência no uso dos benzodiazepínicos, sendo os mais prescritos: o Clonazepam e o Diazepam e um predomínio do uso psicofármacos e psicotrópicos entre três a seis anos, sendo prescritos, em sua maioria, por clínicos gerais, corroborando com a investigação de Passos Neto et al. (2020).

Oliveira et al. (2021) explicitaram que apesar de ter havido aumento no consumo geral de medicamentos sujeitos a controle especial, constatou-se que a taxa de crescimento no uso de Sertralina, Risperidona e Clonazepam superou a taxa de crescimento populacional. E, mais, o Fenobarbital, Sertralina, Fluoxetina e Diazepam foram os medicamentos mais utilizados no município do estudo.

Molck et al. (2021) concluíram que a medicação é a resposta a priori e praticamente exclusiva aos diferentes impasses subjetivos e ao sofrimento psíquico, sem alternativas anteriores à sua prescrição. Em alguns casos foram oferecidos grupos terapêuticos ou psicólogos, como um auxílio ao medicamento.

Identificou-se a medicação como a única forma de produção de cuidado permanente, assinalando que a intervenção de Saúde Mental na APS ficou restrita à prescrição farmacológica, centralizada na figura do médico. As causas psicossociais, experiências de vida e singularidades não tiveram relevância ou foram encaminhadas para outros profissionais, revelando a necessidade de buscar outras formas de respostas à demanda sintomática (Molck, et al., 2021).

Para Rodrigues et al. (2020) a organização de um sistema de saúde capacitado no diagnóstico correto dos transtornos mentais comuns e opções terapêuticas podem impactar no uso e acesso aos psicotrópicos.

A medicalização excessiva dos transtornos de humor, não pode ser descartada e esses podem estar relacionados a problemas sociais e econômicos. Assim, estimar o consumo de antidepressivos e benzodiazepínicos pode ser considerado indicador do cuidado em saúde promovido na APS dos municípios brasileiros (Oliveira et al., 2021).

\section{Conclusões}

A revisão integrativa, realizada na perspectiva da abordagem qualitativa, revela que os artigos não identificam quais são as situações em que os psicofármacos e/ou psicotrópicos são prescritos, como também, não problematizam o perfil das pessoas que os utilizam. Acredita-se que a predominância da abordagem quantitativa dos artigos analisados determinou esse contexto. 
Tendo em vista que os principais prescritores desses medicamentos são clínicos gerais que geralmente atuam em Unidades de Saúde da Família (USF) e Unidades Básicas de Saúde (UBS), aponta-se a necessidade de Educação Continuada e Permanente em Saúde Mental a esses profissionais.

E, mais, deve-se promover a educação em saúde a respeito do uso de psicofármacos e/ou psicotrópicos para usuários e população em geral, visando a compreensão acerca dos efeitos colaterais desses medicamentos como dependência e tolerância.

Sabe-se que os psicofármacos/psicotrópicos são considerados caminho para o enfrentamento dos problemas psíquicos enfrentados na vida. No entanto, considera-se que os profissionais de saúde ponderem o seu uso bem como identifiquem outras formas para as pessoas possam enfrentar sua realidade.

Dessa forma, ressalta-se o uso racional desses medicamentos por meio de políticas públicas que fomente um diagnóstico psiquiátrico preciso bem como uma prescrição e dispensação efetiva. Além da disponibilização de outras formas de tratamento e acompanhamento como terapia com psicólogos, grupos de apoio, entre outros.

\section{Referências}

Baldoni, A. O., Ayres, L. R., Martinez, E. Z., Dewulf, N. L. S., Santos, V, Obreli-Neto, P. R., Pereira, L. R. L. (2013). Pharmacoepidemiological profile and polypharmacy indicators in elderly outpatients. Brazilian Journal of Pharmaceutical Sciences, v. 49, n. 3, p. 443-452.

Bear, M. F., Connors, B. W., Paradiso, M. A. (2010). Neurociências: desvendando o sistema nervoso. 3. ed. Artmed.

Borges, T. L., Hegadoren, K. M., Miasso, A. I. (2015). Transtornos mentais comuns e uso de psicofármacos em mulheres atendidas em unidades básicas de saúde em um centro urbano brasileiro. Revista Pan-americana de Saúde Pública, v. 38, n. 3, p. 195-201.

Borges, T. L., Miasso, A. I., Vedana, K. G. G., Telles Filho, P. C. P., Hegadoren, K. M. (2015). Prevalência do uso de psicotrópicos e fatores associados na atenção primária à saúde. Revista Acta Paulista de Enfermagem, v. 28, n. 4, p. 344-349.

Braga, D. C., Bortolini, S. M., Pereira, T. G., Hildebrando, R. B., Conte, T. A. (2016). Uso de psicotrópicos em um município no meio oeste de Santa Catarina. Journal of the Health Sciences Institute. v. 34, n. 2, p. 108-113.

Bueno, D., Almeida, T. T.,Rocha, B. S. (2017). Prevalência de prescrição de medicamentos potencialmente inapropriados para idosos em uma Unidade de Saúde de Porto Alegre RS. Revista de APS, v. 19, n. 3, p. 370-375.

Ercole, F. F., Melo, S. M., Alcoforado, C. L. G. C. (2014). Revisão Integrativa versus Revisão Sistemática. REME: Revista Mineira de Enfermagem, v. 18, n. 1, p. 9-11.

Filardi, A. F. R., Mendonça, S. A. M., Oliveira, D. R. (2021). O ser humano é assim, sofre, mas alguns dias são piores: A percepção dos pacientes para o início do uso dos medicamentos psicotrópicos. Revista Psicologia em Estudo.

Fontana, A. M. (2005) Manual de clínica em psiquiatria. Editora Atheneu

Ganong, L. H. (1987) Integrative reviews of nursing research. Res. Nurs. Health, v. 10, n. 1, p. 111.

Guerra, C. S., Herculano, M. M., Ferreira Filha, M. O., Dias, M. D., Cordeiro, R. C., Araújo, V. S. (2013). Perfil epidemiológico e prevalência do uso de psicofármacos em uma unidade de referência para saúde mental. Revista de Enfermagem UFPE online, v. 7. n. 6, p. 4444-4451.

Katzung, B. G. (2010). Farmacologia básica e clínica. 10. ed. AMGH.

Lima, A. F. B. S., Fleck, M. P. A. (2011). Qualidade de vida, diagnóstico e tratamento de pacientes com depressão maior: uma coorte prospectiva em cuidados primários. Brazilian Journal of Psychiatry, v. 33, n. 3, p. 245-251. 
Medeiros Filho, J. S. A., Azevedo, D. M., Pinto, T. R., Silva, G. W. S. (2018). Uso de psicofármacos na atenção primária à saúde. Revista Brasileira em Promoção da Saúde, v. 31, n. 3, p. 1-12.

Mezzari, R., Iser, B. P. M. (2015). Desafios na prescrição de benzodiazepínicos em unidades básicas de saúde. Revista AMRIGS, v. 59, n. 3, p.198-203.

Molck, B. V., Barbosa, G. C., Domingos, T. S. (2021). Psicotrópicos e Atenção Primária à Saúde: a subordinação da produção de cuidado à medicalização no contexto da Saúde da Família. Revista Interface: comunicação, saúde e educação.

Nordon, D. G., Akamine, K., Novo, N. F., Hübner, C. K. (2009). Características do uso de benzodiazepínicos por mulheres que buscavam tratamento na atenção primária. Revista de Psiquiatria do Rio Grande do Sul, v. 31, n. 3, p. 152-158.

Oliveira V, M. (2009). Dependencia a benzodiazepinas en un centro de atención primaria de salud: Magnitud del problema y orientaciones para el manejo integral. Revista Chilena de NeuroPsiquiatria, v. 47, n. 2, p. 132-137.

Oliveira, J. R. F., Varallo, F. R., Jirón, M., Ferreira, I. M. L., Siani-Morello, M. R., Lopes, V. D., Pereira, L. R. L. (2021). Descrição do consumo de psicofármacos na atenção primária à saúde de Ribeirão Preto, São Paulo, Brasil. Revista Caderno de Saúde Pública. v.37(1).

Organização Mundial da Saúde. (2006). Neurociência do uso e da dependência de substâncias psicoativas. Roca, p.18-30.

Passos Neto, C. D., Leite, E. S., Martins, A. K. L., Oliveira, F. B., Castro, A. P., Pimenta, C. J. L. (2020). Consumo de benzodiazepínicos por idosos usuários da Estratégia de Saúde da Família. Revista de Pesquisa, Cuidado é Fundamental, v. 12, p. 883-889.

Pereira, M. O., Souza, J. M., Costa, A. M., Vargas, D., Oliveira, M. A. F., Moura, W. N. (2012). Perfil dos usuários de serviços de Saúde Mental no município de Lorena - São Paulo. Acta Paulista de Enfermagem, v. 25, n. 1, p. 48-54.

Prado, M. A. M. B., Francisco, P. M. S. B., Barros, M. B. A. (2017). Uso de medicamentos psicotrópicos em adultos e idosos residentes em Campinas, São Paulo: um estudo transversal de base populacional. Revista Epidemiologia e Serviços de Saúde, v. 26, n. 4, p. 747-758.

Ramon, J. L., Santos, D. A. S., Beltrão, B. L. A., Goulart, L. S., Ribeiro, L. A., Faria, F. R., Olinda, R. A. (2019). Uso de psicotrópicos em uma unidade de Estratégia de Saúde da Família. Revista Enfermagem Atual in Derme, v. 87, n. 25.

Rocha, B. S., Werlang, M. C. (2012). Psicofármacos na Estratégia de Saúde da Família: perfil de utilização, acesso e estratégias para a promoção do uso racional. Ciências \& Saúde Coletiva, v. 18, n. 1, p. 3291-3300.

Rodrigues, P. S., Francisco, P. M. S. B., Fontanella, A. T., Borges, R. B., Costa, K. S. (2020). Uso e fontes de obtenção de psicotrópicos em adultos e idosos brasileiros. Ciência \& Saúde Coletiva, 25(11): p. 4601-4614.

Sadock, B. J.; Sadock, V. A., Sussman, N. (2015). Manual de farmacologia psiquiátrica de Kaplan \& Sadock. 6. ed. Artmed.

Sánchez Ricardo, L. I., Hérnandez Gárciga, F. F. (2010). Consumo de benzodiazepinas en pacientes geriátricos del Consultorio 12, Policlínico "Campo Florido". Revista Cubana de Farmácia, v. 44, n. 3, p. 346-353.

Schallemberger, J. B., Colet, C. F. (2016). Assessment of dependence and anxiety among benzodiazepine users in a provincial municipality in Rio Grande do Sul, Brazil. Trends in Psychiatry and Psychotherapy, v. 38, n. 2, p. 63-70.

Schellack, G. (2006). Farmacologia: uma abordagem didática. Fundamento Educacional.

Schenkel, M., Colet, C. F. (2016). Uso de antidepressivos em um município do Rio Grande do Sul. Arquivos de Ciências da Saúde UNIPAR, v. 20, n. 1, p. 33-42.

Secretaria de Ciência, Tecnologia e Insumos Estratégicos, Ministério da Saúde (2017). Componente avaliação dos serviços de assistência farmacêutica básica: resultados. Ministério da Saúde. 
Silva, V. P., Botti, N. C. L., Oliveira, V. C., Guimarães, E. A. A. (2016). Características do uso e da dependência de benzodiazepínicos entre usuários: atenção primária à saúde. Revista Enfermagem UERJ, v. 24, n. 6.

Silva, V. P., Botti, N. C. L., Oliveira, V. C., Guimarães, E. A. A. (2015). Perfil epidemiológico dos usuários de benzodiazepínicos na atenção primária à saúde. Revista de Enfermagem do Centro Oeste Mineiro, v. 5, n. 1, p. 1393-1400.

Telles Filho, P. C. P., Chagas, A. R., Pinheiro, M. L. P., Lima, A. M. J., Durão, A. M. S. (2011). Utilização de benzodiazepínicos por idosos de uma Estratégia de Saúde da Família: implicações para enfermagem. Escola Anna Nery, v.15, n. 3, p. 581-586.

Videbeck, S. L. (2012). Enfermagem em saúde mental e psiquiatria. 5. ed. Artmed.

Whittemore, R., Knafl, K. (2005) The integrative review: updated methodology. J. Adv. Nurs., v. 52, n. 5 , p. 546-553.

Yoneyama, B. C., Maruiti, A. M. P., Esteves, R. Z. (2016). Um olhar sobre os usuários de medicamentos psicoativos acompanhados na Atenção Primária em Saúde em Maringá Paraná. Revista de Saúde Pública do Paraná Online, v. 17, n. 1, p. 114-120. 